\title{
Ganho genético após uma geração de seleção individual para peso final e variáveis morfométricas em tilápia
}

\author{
Genetic gain after one generation of individual selection for final weight and morphometric \\ variables in tilapias
}

\section{Bruno Corrêa da Silva*, Natália da Costa Marchiori, Fabiano Muller Silva \& Haluko Massago}

Empresa de Pesquisa Agropecuária e Extensão Rural de Santa Catarina, Florianópolis, SC, Brasil. *Autor para correspondência: brunosilva@epagri.sc.gov.br.

\begin{abstract}
RESUMO
Este estudo teve como objetivo avaliar o ganho genético para peso final, e a influência da seleção em outras variáveis zootécnicas e morfométricas, de tilápias-do-nilo selecionadas dentro de diferentes grupos genéticos, após uma geração de seleção individual. Após a seleção, cinco grupos genéticos foram amostrados para realizar o estudo. Foram realizados dez acasalamentos em viveiros de $50 \mathrm{~m}^{2}$, separadamente, para cinco grupos selecionados e cinco não selecionados (peixes amostrados da média de peso dos mesmos grupos genéticos para serem utilizados como controle), totalizando dez viveiros. De cada população foram acasaladas 30 fêmeas com 12 machos, totalizando 300 fêmeas e 120 machos. Após 21 dias, foram coletadas as nuvens, das quais 500 larvas por grupo genético foram amostradas, realizando a alevinagem em 10 tanques de $100 \mathrm{~L}$, separadamente para cada grupo, seguido de recria em dez tanques-rede de $1 \mathrm{~m}^{3}$ dentro de um viveiro de $300 \mathrm{~m}^{2}$. Posteriormente 30 tilápias $(30,0 \pm 1,6 \mathrm{~g})$ de cada grupo genético foram marcadas com transponder magnético, totalizando 300 animais. Estes foram distribuídos em três viveiros de $50 \mathrm{~m}^{2}$, de modo a ficar 10 animais de cada grupo por viveiro. Após 140 dias de cultivo, os animais foram despescados para avaliação. A seleção individual realizada ocasionou um ganho do peso final do cultivo e no peso do filé de $8,4 \%$ e 9,5\%, respectivamente. Além disso, os animais selecionados apresentaram aumento no comprimento total, comprimento padrão, altura corporal, largura corporal e comprimento de tronco. Já outras variáveis como sobrevivência do cultivo, rendimento de carcaça, rendimento de filé e comprimento de cabeça, não foram alterados pela seleção para peso final.
\end{abstract}

PALAVRAS-CHAVE: Oreochromis niloticus, melhoramento genético, ganho de peso, tanque escavado.

\begin{abstract}
Genetic gain for final weight and influence of selective breeding on several zootechnical and morphometric parameters of selected Nile tilapia within different genetic groups, after one generation of individual selection, were evaluated. After selection, five genetic groups were sampled for current analysis. Ten separate matings were carried out in $50 \mathrm{~m}^{2}$ fish ponds for five selected groups and five non-selected groups (sampled fish with mean weight of the same genetic groups for control). Thirty females from each genetic group were mated with twelve males, totaling 300 females and 120 males. Larvae from ponds were collected after 21 days, from which 500 larvae were sampled per genetic group, with hatchery in ten $100 \mathrm{~L}$ tanks, separately for each group, followed by rearing in ten $1 \mathrm{~m}^{3}$ hapas in a $300 \mathrm{~m}^{2}$ fish pond. Afterwards, 30 tilapias $(30.0 \pm 1.6 \mathrm{~g})$ from each genetic group, totaling 300 animals, were tagged with magnetic transponder. These were distributed into three $50 \mathrm{~m}^{2}$ ponds with ten animals of each group per fish pond. After 140 days of rearing, the animals were harvested for evaluation. Individual selection was characterized by final and fillet weight gain, respectively at $8.4 \%$ and $9.5 \%$. In addition, the results showed an increase in total length, standard length, body height, body width and corrected length. However, no modifications occurred in parameters such as survival rate, carcass yield, fillet yield, and head length by final weight selection.
\end{abstract}

KEYWORDS: Oreochromis niloticus, selective breeding, weight gain, fish pond. 


\section{INTRODUÇÃO}

A tilápia-do-nilo, Oreochromis niloticus (Linnaeus, 1758) (Perciformes: Cichlidae), destaca-se como o peixe de maior potencial para a aquicultura continental, haja vista sua rusticidade, crescimento rápido e adaptação ao confinamento (WEBSTER \& LIM 2006). Hoje, esta espécie representa aproximadamente $45,4 \%$ da produção de pescado continental brasileira e $71,2 \%$ da produção catarinense (IBGE 2015, SILVA et al. 2017), crescendo em média 14\% ao ano nos últimos anos (KUBITZA 2015).

Contudo, para a manutenção desse crescimento é necessário um alto padrão genético, evitando problemas causados pela endogamia ao longo das gerações, como diminuição da taxa de crescimento, desuniformidade do lote e deformidades genéticas (GJEDREM 2012, TURRA et al. 2013). Para isto, é necessária a formação de programas de melhoramento genético (GJEDREM 2005).

Segundo GJEDREM (2012), pelo menos metade dos programas de melhoramento genético de tilápia do mundo utiliza como base de sua população, o material derivado da linhagem Gift, do inglês Genetically Improved Farmed Tilapia. Dentre as linhagens de tilápias utilizadas comercialmente no Brasil, esta se destaca não somente pelo seu desempenho, mas também por ser a mais difundida no país (BARROSO et al. 2016). Esta linhagem foi desenvolvida na Ásia, nas instalações de um centro de pesquisa das Filipinas, por meio de uma colaboração internacional. O objetivo inicial do projeto foi aplicar a tecnologia de melhoramento genético conhecida para a melhoria zootécnica de uma espécie de peixe tropical, e a tilápia foi escolhida por possuir um curto intervalo de geração e por sua rusticidade (BENTSEN et al. 2017).

A tilápia Gift foi introduzida no Brasil em 2005 pela antiga Secretaria Especial de Aquicultura e Pesca (SEAP) e a Universidade Estadual de Maringá (UEM) (HALFEN et al. 2012, HILSDORF et al. 2015). Sendo assim, com objetivo de fornecer matrizes de qualidade aos produtores de alevinos de Santa Catarina e dar continuidade ao melhoramento genético da tilápia, considerando as condições climáticas e de cultivo do Estado, a Epagri (Empresa de Pesquisa Agropecuária e Extensão Rural de Santa Catarina) iniciou em 2011 um projeto de seleção individual de tilápias da linhagem Gift.

Entretanto, para avaliar a eficiência de um programa de melhoramento genético torna-se necessário realizar estudos do ganho genético obtido em cada geração de seleção. Para a tilápia-do-nilo, diversos trabalhos vêm mostrando o melhor desempenho em ganho de peso nas linhagens melhoradas (FÜLBER et al. 2010, MASSAGO et al. 2010). A população melhorada da linhagem GST (GenoMar Supreme Tilapia) obteve ganhos genéticos entre 5\% e 15\% por geração (ZIMMERMANN 2003). No Brasil, programas de melhoramento de tilápias da linhagem Gift vêm demonstrando ganhos genéticos importantes ao longo de gerações, em diferentes sistemas de cultivo, como o tanque-rede e o viveiro escavado (OLIVEIRA 2011, OLIVEIRA et al. 2015, PORTO et al. 2015).

Este estudo teve como objetivo avaliar o ganho genético para peso final e a influência da seleção em outras variáveis zootécnicas e morfométricas de tilápias-do-nilo, selecionadas dentro de diferentes grupos genéticos originários de diferentes famílias da UEM, após uma geração de seleção individual.

\section{MATERIAL E MÉTODOS}

\section{Material biológico}

Os animais utilizados no programa de melhoramento da Epagri são indivíduos originários de diferentes famílias do programa de melhoramento genético da UEM, adquiridos nos anos de 2009 e 2013. Durante os anos de 2014 e 2015 foram formados cinco grupos genéticos, a partir de diferentes cruzamentos entre as famílias descritas acima. De cada grupo, foram amostrados aleatoriamente 1.500 indivíduos que foram submetidos a seleção para peso final em viveiros escavados. A seleção ocorreu em viveiros de 300 $\mathrm{m}^{2}$ na unidade de piscicultura da Epagri.

Aos 300 dias de vida, foram selecionados 60 fêmeas e 40 machos com maior peso final em cada grupo, denominados de grupo selecionados. Também foram amostrados animais (60 fêmeas e 40 machos) que estavam na média de peso em cada grupo, e estes foram denominados de grupo controle. Sendo assim, foram formados dez grupos, cinco grupos selecionados e cinco grupos controle.

Neste estudo foram utilizados a prole destes dez grupos genéticos, obtidos através de reprodução durante $\mathrm{o}$ ano de 2016. Para isto, 500 larvas de cada um dos dez grupos genéticos (selecionados e controle) foram amostradas aleatoriamente e povoadas em dez caixas de $100 \mathrm{~L}$, com aeração e renovação, para realizar a alevinagem. Após 28 dias de alimentação com ração contendo $60 \mathrm{mg} \mathrm{kg}^{-1}$ do hormônio 17- $\alpha$ metil-testosterona, os alevinos de cada grupo foram transferidos para dez tanques-rede de $1 \mathrm{~m}^{3}$, dentro de um viveiro de $300 \mathrm{~m}^{2}$, para a fase de recria até atingirem $30 \mathrm{~g}$.

\section{Delineamento experimental}

O experimento foi conduzido de janeiro a junho de 2017. Para isto, 30 espécimes $(30,0 \pm 1,6 \mathrm{~g})$ de 
cada um dos dez grupos (selecionados e controle) foram amostrados aleatoriamente e marcados com transponder magnético, totalizando 300 peixes chipados. Após a marcação, estes foram transferidos para três viveiros de $50 \mathrm{~m}^{2}$, de forma que cada grupo contivesse dez animais em cada viveiro, onde cada viveiro representou uma réplica de cada tratamento. Sendo assim, o experimento foi conduzido com delineamento fatorial (2x5) em blocos, onde o fator 1 eram os animais selecionados e não selecionados, fator 2 eram os diferentes grupos genéticos (G1, G2, G3, G4 e G5) e os blocos eram os diferentes viveiros.

\section{Análises realizadas}

O monitoramento da qualidade de água dos viveiros foi realizado semanalmente. A temperatura, oxigênio e $\mathrm{pH}$ foram monitorados no início da manhã e final de tarde com auxílio de oxímetro digital (Alfakit AT-155) e com pHmetro digital (YSI pH100A), respectivamente. A transparência foi mensurada com disco de Secchi, a alcalinidade por titulação com kit colorímetro (Alfakit ${ }^{\circledR}$ ) e amônia total e nitrito com auxílio do fotocolorímetro microprocessado com kit colorimétrico (Alfakit ${ }^{\circledR}$ ) utilizando os métodos de Nessler e alfanaftilamina, respectivamente. Quinzenalmente, também eram realizadas biometrias com 10 a $20 \%$ dos peixes do viveiro para acompanhamento do crescimento e ajuste da ração, segundo a tabela de alimentação da Epagri (SILVA \& MARCHIORI 2018).

Após 140 dias de cultivo, os viveiros foram despescados para avaliação das variáveis zootécnicas, morfométricas, rendimento de carcaça e filé. Para isto, os peixes foram insensibilizados no gelo e, posteriormente, realizada a leitura do microchip, a medição das variáveis morfométricas (comprimento total, comprimento padrão, altura, largura e comprimento de cabeça) como indicado por OLIVEIRA (2011), além do peso total, peso da carcaça e peso do filé. Para a avaliação do rendimento da carcaça e do filé foram utilizadas as mesmas pessoas para cada processo (limpeza do peixe, filetagem e retirada do couro), com objetivo de padronização dos dados, evitando assim a variância que há na obtenção destas variáveis quando realizado por diferentes pessoas. Além disso, foi obtido o comprimento do tronco através da subtração do comprimento padrão e do comprimento da cabeça (LEONHARDT et al. 2006). A sobrevivência foi calculada usando a média entre os três viveiros para cada grupo genético, separadamente, através da fórmula abaixo:

$$
\text { Sobrevivência }(\%)=100 \times\left(\frac{\text { número de peixes povoados }}{\text { número de peixe despescado }}\right)
$$

\section{Análise estatística}

Os dados finais foram submetidos a análise de Levene e Shapiro-Wilk para avaliar a homocedasticidade e normalidade dos dados. Posteriormente, estes foram submetidos a análise de variância fatorial $(2 \times 5)$ em blocos. Se necessário, a separação de médias foi realizada pelo teste de Tukey. Todas as análises levaram em consideração um nível de significância de 5\%.

\section{RESULTADOS}

A temperatura variou de 18,0 a $28,9^{\circ} \mathrm{C}$ pela manhã e 20,2 a $32,0^{\circ} \mathrm{C}$ no período da tarde. $\mathrm{O}$ oxigênio dissolvido também apresentou uma variação entre os diferentes períodos do dia, com valores entre $3,4 \mathrm{e}$ $7,8 \mathrm{mg} \mathrm{L}^{-1}$ pela manhã e 6,3 a 14,3 $\mathrm{mg} \mathrm{L}^{-1}$ à tarde. $\mathrm{O} \mathrm{pH}$ variou de 6,5 a 7,8 pela manhã, e 7,0 a 10,0 à tarde. Os viveiros apresentaram valores de transparência entre 12 a $35 \mathrm{~cm}$, já a alcalinidade ficou entre $22 \mathrm{e}$ $50 \mathrm{mg} \mathrm{CaCO} \mathrm{L}^{-1}$. A amônia tóxica obteve valores máximos de $0,9 \mathrm{mg} \mathrm{L}^{-1}$ de $\mathrm{NH}_{3}$, enquanto que o nitrito apresentou valor máximo de $0,2 \mathrm{mg} \mathrm{L}^{-1}$ de $\mathrm{NO}_{2}$ (Figura 1).

A prole dos animais selecionados em uma geração de seleção individual apresentou maior peso final e peso de filé em relação a prole dos animais dos grupos controle $(p<0,05)$. Já a sobrevivência, o peso de carcaça, a porcentagem de carcaça e filé não apresentaram diferenças significativas entre a prole dos animais selecionados e dos animais dos grupos controle $(p>0,05)$. Entre os diferentes grupos genéticos, 0 grupo 5 (G5) apresentou menor peso final em relação as demais $(p<0,05)$, contudo, os animais selecionados desse grupo apresentaram crescimento superior aos animais do controle deste mesmo grupo $(p<0,05)($ Tabela 1$)$.

Os animais selecionados apresentaram um aumento no comprimento total, comprimento padrão, altura corporal, largura corporal e comprimento de tronco de 2,4\%, 2,4\%, 3,2\%, 2,7\% e 2,6\%, respectivamente, em relação aos peixes do grupo controle $(p<0,05)$. $O$ comprimento da cabeça não apresentou diferenças significativas entre esses dois grupos $(p>0,05)$. Já entre os grupos genéticos, o $G 2$ apresentou maior comprimento total, comprimento padrão, comprimento de cabeça, altura corporal e comprimento de tronco em relação ao $G 5(p<0,05)$. A largura corporal não variou entre os diferentes grupos genéticos $(p>0,05)$ (Tabela 2). 


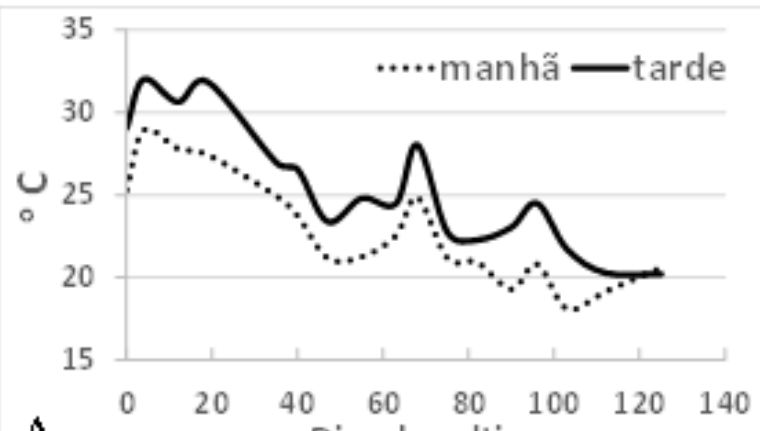

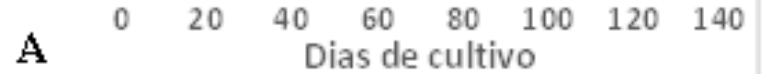
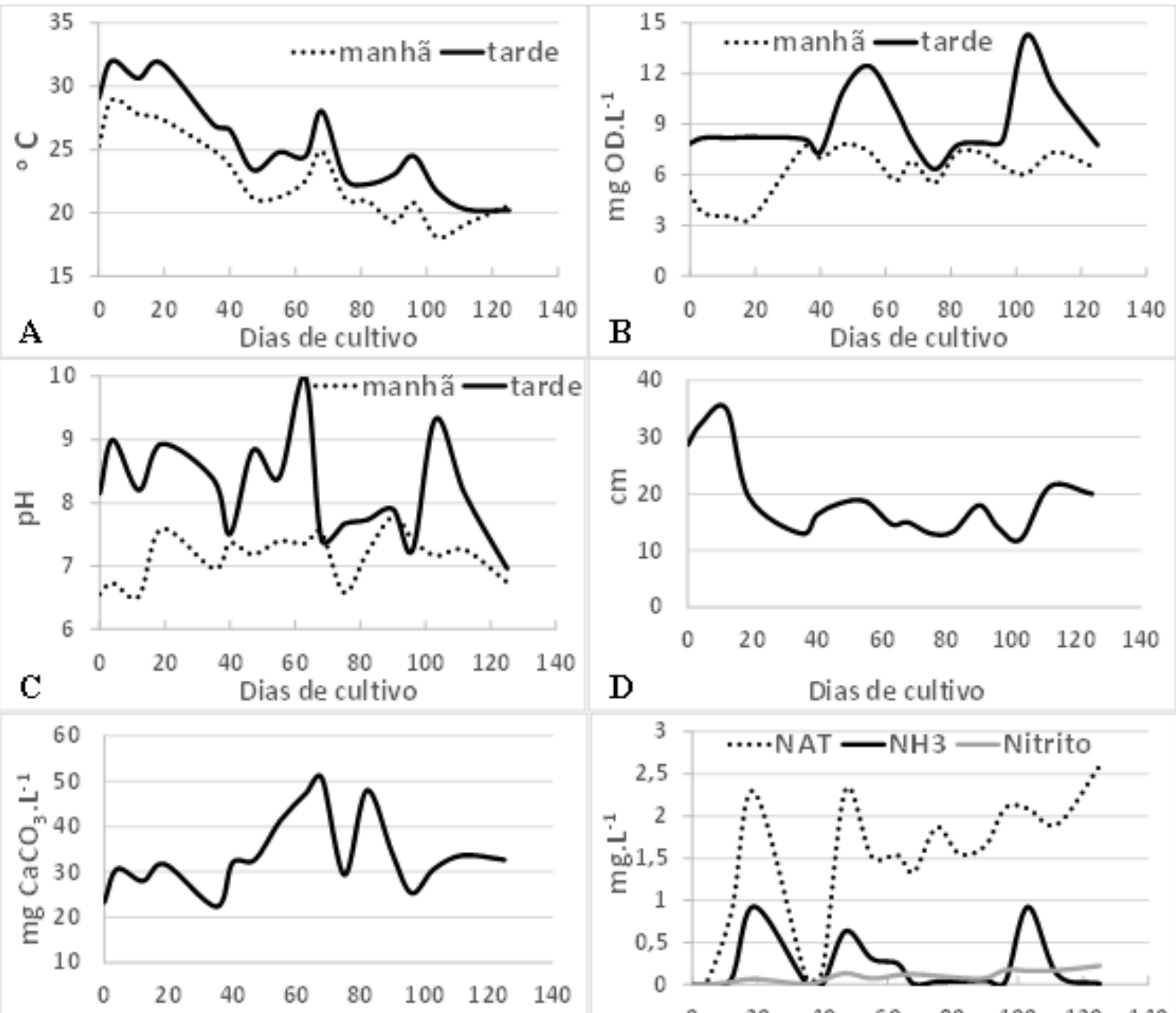

E

Dias de cultivo

D Dias de cultivo

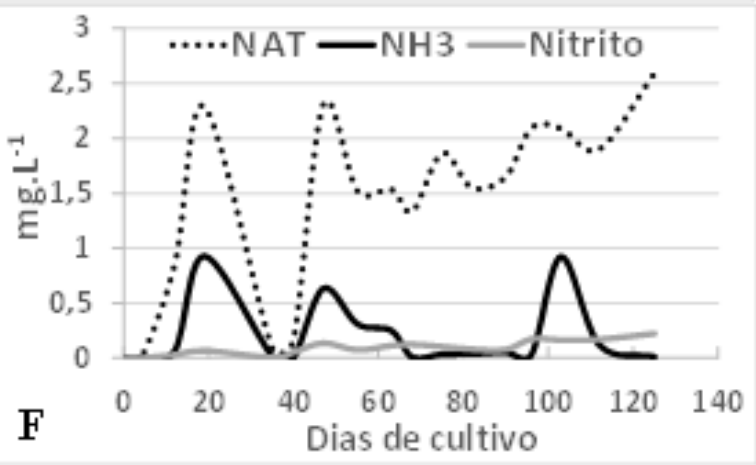

Figura 1. Qualidade de água dos viveiros experimentais para avaliação do ganho genético de tilápias após uma geração de seleção individual. $A=$ Temperatura $\left({ }^{\circ} \mathrm{C}\right), \mathrm{B}=$ Oxigênio dissolvido $(\mathrm{OD})$ pela manhã e tarde $\left(\mathrm{mg} \mathrm{L}^{-1}\right), \mathrm{C}=\mathrm{pH}$ pela manhã e tarde, $\mathrm{D}=$ transparência $(\mathrm{cm}), \mathrm{E}=$ Alcalinidade ( $\left.m g \mathrm{CaCO}_{3} \mathrm{~L}^{-1}\right), \mathrm{F}=$ Nitrogênio amoniacal total (NAT), amônia tóxica $\left(\mathrm{NH}_{3}\right)$ e nitrito.

Figure 1. Water quality of the experimental nurseries to evaluate the genetic gain of tilapia after one individual selection generation. $A=$ Temperature $\left({ }^{\circ} \mathrm{C}\right), B=$ Dissolved Oxygen $(D O)$ for morning and afternoon $\left(\mathrm{mg} \mathrm{L}^{-1}\right), C=\mathrm{pH}$ for morning and afternoon, $D=$ transparency $(\mathrm{cm}), E=$ Alkalinity (mg $\left.\mathrm{CaCO}_{3} \mathrm{~L}^{-1}\right), \mathrm{F}=$ Total Ammoniacal Nitrogen (NAT), toxic ammonia $\left(\mathrm{NH}_{3}\right)$ and nitrite.

Tabela 1. Variáveis zootécnicos da prole de diferentes grupos genéticos de tilápias-do-nilo, linhagem GiftEpagri, após uma geração de seleção individual (média \pm desvio padrão).

Table 1. Zootechnical variables of offspring from different genetic groups of Nile tilapia, of the Gift-Epagri line, after one generation of individual selection (mean \pm standard deviation).

\begin{tabular}{llllccc}
\hline Tratamento & $\begin{array}{c}\text { Peso final } \\
(\mathrm{g})\end{array}$ & $\begin{array}{c}\text { Peso Carcaça } \\
(\mathrm{g})\end{array}$ & $\begin{array}{c}\text { Peso Filé } \\
(\mathrm{g})\end{array}$ & $\begin{array}{c}\text { Carcaça } \\
(\%)\end{array}$ & $\begin{array}{c}\text { Filé } \\
(\%)\end{array}$ & $\begin{array}{c}\text { Sobrevivência } \\
(\%)\end{array}$ \\
\hline G1 & $398,5 \pm 13,2^{\mathrm{a}}$ & $256,0 \pm 9,2$ & $124,3 \pm 3,7$ & $64,1 \pm 0,9$ & $31,5 \pm 0,2$ & $93,3 \pm 2,9$ \\
G2 & $409,1 \pm 12,6^{\mathrm{a}}$ & $260,0 \pm 16,1$ & $125,6 \pm 4,1$ & $64,4 \pm 0,2$ & $30,4 \pm 0,4$ & $93,3 \pm 7,6$ \\
G3 & $393,0 \pm 13,2^{\mathrm{a}}$ & $253,3 \pm 10,6$ & $122,6 \pm 4,9$ & $65,2 \pm 0,5$ & $30,8 \pm 0,4$ & $95,0 \pm 5,0$ \\
G4 & $395,1 \pm 6,4^{\mathrm{a}}$ & $253,2 \pm 6,0$ & $122,8 \pm 3,7$ & $64,2 \pm 0,4$ & $31,0 \pm 0,2$ & $96,7 \pm 2,9$ \\
G5 & $345,3 \pm 10,3^{\mathrm{b}}$ & $217,7 \pm 6,8$ & $110,2 \pm 4,8$ & $64,3 \pm 0,8$ & $31,4 \pm 0,3$ & $96,7 \pm 2,9$ \\
\hline Controle & $372,6 \pm 8,0^{\mathrm{B}}$ & $240,3 \pm 6,3$ & $115,6 \pm 2,3^{\mathrm{B}}$ & $64,5 \pm 0,4$ & $31,0 \pm 0,2$ & $94,0 \pm 2,0$ \\
Selecionados & $403,8 \pm 8,1^{\mathrm{A}}$ & $255,9 \pm 7,9$ & $126,6 \pm 2,8^{\mathrm{A}}$ & $64,3 \pm 0,4$ & $31,1 \pm 0,2$ & $96,0 \pm 0,0$ \\
\hline
\end{tabular}

Diferentes letras significam diferenças estatísticas no teste de Tukey $(p<0,05)$. Letras minúsculas - diferenças entre os grupos genéticos. Letras maiúsculas - diferenças entre os animais selecionados e o grupo controle. 
Tabela 2. Variáveis morfológicas da prole de diferentes grupos genéticos de tilápias-do-nilo, linhagem GiftEpagri, após uma geração de seleção individual (média \pm desvio padrão).

Table 2. Morphological variables of offspring from different genetic groups of Nile tilapia, of Gift-Epagri line, after one generation of individual selection (mean \pm standard deviation).

\begin{tabular}{|c|c|c|c|c|c|c|}
\hline Tratamento & $\begin{array}{l}\mathrm{CT} \\
(\mathrm{cm})\end{array}$ & $\begin{array}{l}\mathrm{CP} \\
(\mathrm{cm})\end{array}$ & $\begin{array}{l}\mathrm{CC} \\
(\mathrm{cm})\end{array}$ & $\begin{array}{l}\mathrm{AC} \\
(\mathrm{cm})\end{array}$ & $\begin{array}{l}\text { LC } \\
(\mathrm{cm})\end{array}$ & $\begin{array}{l}\text { CTr } \\
\text { (cm) }\end{array}$ \\
\hline$\overline{\mathrm{G} 1}$ & $26,2 \pm 0,2^{\mathrm{ab}}$ & $21,8 \pm 0,1$ ab & $6,0 \pm 0,1^{a b}$ & $9,5 \pm 0,1^{a}$ & $4,5 \pm 0,1$ & $15,9 \pm 0,1^{a b}$ \\
\hline G2 & $26,8 \pm 0,2^{a}$ & $22,3 \pm 0,2^{a}$ & $6,2 \pm 0,1^{a}$ & $9,6 \pm 0,1^{a}$ & $4,5 \pm 0,1$ & $16,1 \pm 0,1^{a}$ \\
\hline G3 & $25,8 \pm 0,3^{b c}$ & $21,5 \pm 0,3^{b}$ & $6,1 \pm 0,1^{a b}$ & $9,6 \pm 0,1^{a}$ & $4,4 \pm 0,1$ & $15,4 \pm 0,2^{b c}$ \\
\hline G4 & $26,1 \pm 0,2^{a b}$ & $21,7 \pm 0,1 \mathrm{ab}$ & $6,0 \pm 0,1 \mathrm{ab}$ & $9,6 \pm 0,1^{a}$ & $4,4 \pm 0,1$ & $15,7 \pm 0,1 \mathrm{ab}$ \\
\hline G5 & $25,0 \pm 0,2^{c}$ & $20,8 \pm 0,2^{c}$ & $5,9 \pm 0,1^{b}$ & $9,1 \pm 0,1^{b}$ & $4,3 \pm 0,1$ & $14,9 \pm 0,2^{c}$ \\
\hline Controle & $25,7 \pm 0,3^{\mathrm{B}}$ & $21,4 \pm 0,3^{\mathrm{B}}$ & $6,0 \pm 0,1$ & $9,3 \pm 0,1^{\mathrm{B}}$ & $4,3 \pm 0,1^{\mathrm{B}}$ & $15,4 \pm 0,3^{\mathrm{B}}$ \\
\hline Selecionados & $26,3 \pm 0,3^{A}$ & $21,9 \pm 0,2^{A}$ & $6,1 \pm 0,1$ & $9,6 \pm 0,1^{\mathrm{A}}$ & $4,5 \pm 0,1^{\mathrm{A}}$ & $15,8 \pm 0,1^{\mathrm{A}}$ \\
\hline
\end{tabular}

Diferentes letras significam diferenças estatísticas no teste de Tukey $(p<0,05)$. Letras minúsculas indicam diferenças entre as populações. Letras maiúsculas indicam diferenças entre os animais selecionados e o grupo controle. $\mathrm{CT}=$ comprimento total; $\mathrm{CP}=$ comprimento padrão; $C C=$ comprimento da cabeça; $A C=$ altura corporal; $L C=$ largura corporal; $C T r=$ comprimento do tronco.

\section{DISCUSSÃO}

Durante quase todo o cultivo, as variáveis de qualidade de água mantiveram-se dentro da faixa considerada adequada para a tilápia-do-nilo, conforme KUBITZA (2011). Porém, a temperatura apresentou valores abaixo de $25 \stackrel{\circ}{\circ}$ durante os últimos dois meses de cultivo, assim como amônia e a alcalinidade que, em alguns casos pontuais, apresentaram valores inadequados, como amônia tóxica $\left(\mathrm{NH}_{3}\right)$ acima de $0,5 \mathrm{mg} \mathrm{L}^{-1} \mathrm{e}$ alcalinidade abaixo de $30 \mathrm{mg} \mathrm{CaCO}_{3} \mathrm{~L}^{-1}$. A transparência se manteve após o primeiro mês de cultivo na sua maioria das vezes abaixo dos $20 \mathrm{~cm}$, reflexo de um excesso de floração de algas no viveiro que, por sua vez, podem resultar em uma maior variação diária do pH e oxigênio. Contudo, devido a boa alcalinidade e adequado uso do aerador, $\mathrm{o} \mathrm{pH}$ ao final da tarde e o oxigênio ao início da manhã se mantiveram adequados.

A água dos viveiros foi renovada em 30\% quando apresentou transparência abaixo de $20 \mathrm{~cm}$ ou amônia tóxica $\left(\mathrm{NH}_{3}\right)$ acima de $0,5 \mathrm{mg} \mathrm{L}^{-1}$. Enquanto que nas semanas em que a alcalinidade da água dos viveiros apresentou valores inferiores a $30 \mathrm{mg} \mathrm{CaCO}_{3} \mathrm{~L}^{-1}$, esta foi corrigida com adição de $100 \mathrm{~g} \mathrm{~m}^{2}$ de calcário agrícola.

Segundo os resultados zootécnicos obtidos neste estudo, a seleção individual de tilápia-do-nilo da linhagem Gift, através do programa de melhoramento de tilápia da Epagri para Santa Catarina, ocasionou em uma geração de seleção, o ganho de $8,4 \%$ para o peso final e $9,5 \%$ para peso de filé. Além disso, não foi observado em animais selecionados, a redução nos parâmetros de sobrevivência, rendimento de filé e rendimento de carcaça, parâmetros importantes para indústria de beneficiamento, principal destino de venda dos produtores de tilápia (BARROSO et al. 2017).

BENTSEN et al. (2017) relataram os resultados obtidos nos primeiros anos do projeto que desenvolveu a linhagem Gift. $O$ projeto obteve um ganho acumulado para ganho de peso nas primeiras cinco gerações de $88 \%$, em relação a população base, com ganhos por gerações variando de 9 a $20 \%$. Para o Brasil foram trazidas a $15^{\circ}$ geração de seleção em 2005, e deu-se continuidade com o melhoramento através dos estudos relatados a seguir pelo grupo da Universidade Estadual de Maringá (UEM).

No programa de melhoramento genético de tilápia Gift da UEM após quatro anos de cruzamento (quatro gerações), a população obteve um ganho acumulado de $28 \%$ em viveiros escavados, ou seja, obteve um ganho médio de 7\% por geração (OLIVEIRA 2011). Em outro estudo, também com a tilápia Gift oriunda da UEM, em quatro gerações de seleção para o cultivo em tanque-rede, o ganho genético foi de $15 \%$, média de $3,8 \%$ por geração (OLIVEIRA et al. 2015). Outro estudo mostrou uma média de ganho genético da tilápia por geração de seleção de 7,5\% (5,1 a 9,4\%) (PORTO et al. 2015).

Acredita-se que a seleção realizada neste estudo seja aproximadamente a $20^{\circ}$ geração deste material, desde o início do projeto Gift no final dos anos 80, e ainda é possível observar ganhos significativos a cada geração, mostrando a capacidade que este material ainda tem de continuar sendo selecionado e melhorado. PONZONI et al. (2011) relatam que após a difusão da linhagem Gift para outros lugares do mundo no final dos anos 90, ainda foi possível acompanhar ganhos genéticos entre 10 a $15 \%$ por geração por pelo menos mais 6 gerações.

Com dados de apenas uma geração de seleção de tilápia-do-nilo, SANTOS et al. (2011) obtiveram um ganho genético para peso da despesca de $5 \%$ e indiretamente um aumento na sobrevivência de $2 \%$. Já neste estudo, apesar do ganho do peso dos animais selecionados em uma geração ser em média 8,4\%, a 
sobrevivência não mostrou alterações significativas, corroborando com o relato de outros autores que mostram dados de um programa na Malásia com oito gerações de seleção da tilápia-do-nilo (2002 a 2009), também derivadas do programa Gift, no qual apesar de um ganho acumulado para o peso de $64 \%$, a sobrevivência não sofreu alterações (PONZONI et al. 2011). Esta informação é de grande valor para o programa de melhoramento genético, já que em muitos casos para peixes, a resistência a doenças ou ao estresse podem apresentar uma correlação negativa com o ganho de peso, ou seja, os animais selecionados para ganho de peso podem ser animais com menor resistência a doenças, e consequentemente, possuir uma menor sobrevivência (GJEDREM 2005). Contudo, EKNATH et al. (1998) relataram uma correlação positiva, porém muito baixa $(0,2)$, entre o peso de despesca e a sobrevivência para a tilápia, o que pode ajudar a explicar os nossos resultados e os de PONZONI et al. (2011), relatados acima.

Comparando diferentes linhagens de tilápia-do-nilo, MASSAGO et al. (2010) e FÜLBER et al. (2010) relataram que linhagens Gift, apresentaram maior ganho de peso sobre as linhagens Bouaké e Chitralada não melhoradas, atingindo uma diferença de crescimento acima dos $20 \%$ entre as linhagens. Estes destacam a importância do melhoramento genético para aumentar a produtividade da tilapicultura nacional.

MARCOS et al. (2016) observaram que de sete famílias de tambaqui (Colossoma macropomum) selecionadas para ganho de peso, apenas cinco apresentaram ganho genético $(+14,8 \%$ em média) após a realização de um ensaio semelhante a este trabalho, contudo em nosso estudo todas os grupos selecionados apresentaram ganho de peso superior aos não selecionados. Além disso, quatro famílias de tambaqui apresentaram ganhos morfométricos (comprimento total, comprimento padrão, comprimento de cabeça, largura e altura corporal) em relação ao grupo controle (MARCOS et al. 2016), corroborando com o resultado do nosso estudo, que mostrou que a seleção para peso final pode trazer ganhos nas características morfométricas. Outro estudo com tilápia-do-nilo apresentou resultados que comprovaram que a seleção para ganho de peso diário ocasionou em três gerações ganhos indiretos para largura corporal, 1,11 a 3,08\% por geração, altura corporal, 1,23 a 3,48\% por geração, e comprimento do tronco, 0,89 a 2,56\% por geração (PORTO et al. 2015).

Além disso, outros autores também observaram diferenças nos padrões morfométricos de animais submetidos a melhoramento genético por seleção ou entre diferentes linhagens. ALLAMAN et al. (2013) observaram diferentes relações morfométricas de tilápias com diferentes origens e, dentre elas, a linhagem Gift obteve maior peso, maior altura e largura comparado a outras populações. BOSCOLO et al. (2001) e LEONHARDT et al. (2006) observaram que a linhagem com maior desempenho zootécnico também apresentava diferenças morfométricas em relação as demais. Isto se deve pelo fato dos parâmetros morfológicos estarem fortemente correlacionados com o peso. OLIVEIRA et al. (2014) e SILVA et al. (2018) observaram correlações acima de 0,85 entre o peso corporal da tilápia-do-nilo e seus parâmetros morfológicos. Sendo assim, quando o animal é selecionado para ganho de peso, indiretamente se está selecionando animais com outro padrão morfológico.

Segundo BENTSEN et al. (1998), dificilmente os produtores familiares de alevinos de tilápia tem estrutura e recurso para manter um programa de melhoramento genético por seleção como deve ser feito, por isso, deveriam todo início de safra procurar centrais de seleção para repor suas matrizes e assim, evitar perdas por endogamia e explorar a heterose provenientes das centrais de seleção. Esse foi o objetivo inicial do desenvolvimento da linhagem Gift, e é o objetivo da unidade de melhoramento genético da Epagri, disponibilizar aos produtores rurais de alevino de Santa Catarina e do sul do Brasil matrizes com qualidade genética, para que o produtor que realize a engorda possua um melhor resultado a campo.

Desde o início do projeto de melhoramento da linhagem Gift, em 1988, até 27 anos depois, em 2015, a produção de tilápia no mundo passou de 127 mil ton para 3,93 milhões de ton, um aumento de quase 31 vezes (FAO 2017). No Brasil, a produção de tilápia em 2005, ano da introdução desta linhagem, foi de 67,8 mil ton. Já em 2015, após dez anos, a produção foi de 219,4 mil ton, representando um crescimento de 223\% (IBGE 2015). Estes dados confirmam o que foi dito anteriormente, que para se manter o crescimento da atividade é importante a manutenção e melhoria da qualidade genética das tilápias produzidas comercialmente. Sendo assim, o desenvolvimento de linhagens mais adaptadas as condições de cultivo de cada região, poderão otimizar o potencial de cultivo destes locais e manter o crescimento da atividade.

\section{CONCLUSÃO}

Com este estudo foi possível concluir que a seleção individual realizada ocasionou um ganho do peso final do cultivo e no peso do filé de $8,4 \%$ e $9,5 \%$, respectivamente. Além disso, os animais selecionados apresentaram aumento no comprimento total, comprimento padrão, altura corporal, largura corporal e 
comprimento de tronco. Já outros parâmetros como sobrevivência do cultivo, rendimento de carcaça, rendimento de filé e comprimento de cabeça não foram alteradas pela seleção para peso final.

\section{AGRADECIMENTOS}

Os autores agradecem o apoio financeiro do Conselho Nacional de Desenvolvimento Científico e Tecnológico (CNPq) através do financiamento do projeto intitulado "Variabilidade genética de tilápia do Nilo (Oreochromis niloticus L.), linhagem GIFT, e seleção individual dentro de famílias para formação de matrizes (CNPq - CNPq 444577/2014-9)". Além disso, os autores agradecem o suporte financeiro para unidade da Fundação de Amparo à Pesquisa e Inovação do Estado de Santa Catarina (FAPESC) e o suporte técnico no trabalho de Silvano Garcia, João Burg, Leandro Bortoli e Silvio Demarch Filho.

\section{REFERÊNCIAS}

ALLAMAN IB et al. 2013. Weight and morphometric growth of different strains of tilapia (Oreochromis sp). Revista Brasileira de Zootecnia 42: 305-311.

BARROSO RM et al. 2016. Gerenciamento genético da tilápia nos cultivos comerciais. Palmas: EMBRAPA. 64p.

BARROSO RM et al. 2017. Informativo Técnico: Mercado da Tilápia 10. Palmas: EMBRAPA. 6p. (Informativo Técnico).

BENTSEN HB et al. 1998. Genetic improvement of farmed tilapias: growth performance in a complete diallel cross experiment with eight strains of Oreochromis niloticus. Aquaculture 160: 145-173.

BENTSEN HB et al. 2017.Genetic improvement of farmed tilapias: Response to five generations of selection for increased body weight at harvest in Oreochromis niloticus and the further impact of the project. Aquaculture 468: 206217.

BOSCOLO WR et al. 2001. Desempenho e Características de Carcaça de Machos Revertidos de Tilápias do Nilo (Oreochromis niloticus), Linhagens Tailandesa e Comum, nas Fases Inicial e de Crescimento. Revista Brasileira de Zootecnia 30: 1391-1396.

EKNATH AE et al. 1998. Selective breeding of nile tilapia for Asia. In: 6th World Congress on Genetics Applied to Livestock Production. Australia: University of New England Armidale 27: 89-96.

FAO. 2017. Food and Agriculture Organization of the United Nations. Fishery and Aquaculture Statistics, 2015 Roma: FAO Yearbook. 107p.

FÜLBER VM et al. 2010. Desempenho produtivo de três linhagens de tilápia-do-Nilo (Oreochromis niloticus) alimentadas com dois níveis de proteína. Acta Scientiarum. Animal Sciences 32: 77-83.

GJEDREM T. (Ed.) 2005. Selection and breeding programs in aquaculture. Dordrecht: Springer. 364p.

GJEDREM T. 2012. Genetic improvement for the development of efficient global aquaculture: a personal opinion review. Aquaculture 344: 12-22.

HALFEN GE et al. 2012. Caracterização molecular de plantéis de tilápia do Nilo (Oreochromis niloticus L.) em Santa Catarina, Brasil. Journal of Biotechnology and Biodiversity 3: 21-29.

HILSDORF AWS et al. 2015. Desafios do melhoramento genético de organismos aquáticos. Panorama da Aquicultura 147: 36-43.

IBGE. 2015. Instituto Brasileiro de Geografia e Estatística. Produção da Pecuária Municipal 2015. Rio de Janeiro IBGE. 43: $1-49$.

KUBITZA F. 2011. Tilápia: Tecnologia e planejamento na produção comercial. 2.ed. Jundiaí: Acqua Supre Com. Suprim. Aqüicultura. 316p.

KUBITZA F. 2015. Aquicultura no Brasil: principais espécies, áreas de cultivo, rações, fatores limitantes e desafios. Panorama da Aquicultura 150: 10-23.

LEONHARDT JH et al. 2006. Características morfométricas, rendimento e composição do filé de tilápia do Nilo, Oreochromis niloticus, da linhagem tailandesa, local e do cruzamento de ambas. Semina: Ciências Agrárias 27: 125132.

MARCOS R et al. 2016. Weight gain and morphometric growth of genetically improved tambaqui (Colossoma macropomum). Semina: Ciências Agrárias 37: 2521-2527.

MASSAGO H et al. 2010. Growth performance of four strains of tilapia Oreochromis niloticus. Revista Acadêmica Ciências Agrárias e Ambientais 8: 397-403.

OLIVEIRA SN. 2011. Parâmetros genéticos para características de desempenho e morfométricas em tilápias do nilo (Oreochromis niloticus). Dissertação (Mestrado em Zootecnia). Maringá: UEM. 45p.

OLIVEIRA SN et al. 2014. Genetic parameters and morphometric characteristics of two generations from the GIFT strain of the Nile Tilapia. Semina: Ciências Agrárias 35: 3457-3468.

OLIVEIRA CAL et al. 2015. Avaliação genética de tilápias-do-nilo durante cinco anos de seleção. Pesquisa Agropecuária Brasileira 50: 871-877.

PONZONI RW et al. 2011. Genetic improvement of Nile tilapia (Oreochromis niloticus) with special reference to the work conducted by the WorldFish Center with the GIFT strain. Reviews in Aquaculture 3: 27-41.

PORTO EP et al. 2015. Respostas à seleção de características de desempenho em tilápia-do-nilo. Pesquisa Agropecuária Brasileira 50: 745-752.

SANTOS Al et al. 2011. Bayesian genetic parameters for body weight and survival of Nile tilapia farmed in Brazil. 
Pesquisa Agropecuária Brasileira 46: 33-43.

SILVA BC et al. 2017. Desempenho da piscicultura catarinense em 2015. Florianópolis: EPAGRI. 17p. (Documentos 268).

SILVA BC et al. 2018. Morphometric measurements and phenotypic correlations of the tilapia Gift (Oreochromis niloticus) after individual selection. Revista Agropecuária Catarinense 31: 38-40.

SILVA BC \& MARCHIORI NC. 2018. Importância do Manejo Alimentar da Criação da Tilápia. Florianópolis: Epagri. 16p. (Informativo Técnico).

TURRA EM et al. 2013. Ferramentas para o melhoramento genético de peixes em água interiores. X Simpósio Brasileiro de Melhoramento Animal. Uberaba: SBMA. 12p.

WEBSTER CD \& LIM C (Ed.). 2006. Tilapia: biology, culture, and nutrition. New York: The Haworth Press. 704p.

ZIMMERMANN S. 2003. Um moderno instrumental genético no melhoramento e na rastreabilidade de tilápias nilóticas. Panorama da Aquicultura 76: 69-71. 